\title{
ANALYTIC FUNCTIONS OF A PRESPECTRAL OPERATOR
}

\author{
by S. AL-KHEZI
}

(Received 22 February, 1981)

The purpose of this note is to present a unified treatment of the material contained in Chapter 10 of [2] on roots and logarithms of prespectral operators. Our main result gives a sufficient condition for an analytic function of a prespectral operator of class $\Gamma$ to be prespectral of class $\Gamma$. A result in the opposite direction for spectral operators has been obtained by Apostol [1]. Terminology and notation in this paper are as in [2].

I should like to thank my research supervisor Dr. H. R. Dowson for his guidance and encouragement, and the University of Riyadh for their financial support, during the period of research 1977-1981.

The main result of the paper follows. Throughout $X$ is a complex Banach space and $X \neq\{0\}$.

THEOREM 1. Let $A$ be a prespectral operator on $X$ with resolution of the identity $E(\cdot)$ of class $\Gamma$. Let $f$ be a function analytic on a region $\Omega$, such that $\sigma(A) \subseteq f(\Omega)$ and $f^{\prime}(\lambda) \neq 0$ for all $\lambda$ in $\Omega$. Then there is an operator $T$ on $X$ such that $T$ is prespectral of class $\Gamma, \sigma(T) \subseteq \Omega$ and $f(T)=A$.

Proof. Let $\lambda \in \sigma(A)$. Then there is a point $\zeta$ in $\Omega$ such that $f(\zeta)=\lambda$. By Theorem 10.34 of [3,p.217], there exist open neighbourhoods $V_{\zeta}$ and $W_{\lambda}$ such that $f$ is a one-to-one mapping of $V_{\zeta}$ onto $W_{\lambda}$. The set $W_{\lambda}$ is open and $\lambda \in W_{\lambda}$. Hence we can find an open disc $D_{\lambda}$ which is properly contained in $W_{\lambda}$ and has $\lambda$ as its centre. Let $\delta(\lambda)$ be the open disc with centre $\lambda$ and radius half that of $D_{\lambda}$. As $\lambda$ runs through $\sigma(A)$, the corresponding discs

$$
\{\delta(\lambda): \lambda \in \sigma(A)\}
$$

cover $\sigma(A)$. Since $\sigma(A)$ is compact, there is a finite subcovering; that is

$$
\sigma(A) \subseteq \bigcup_{r=1}^{n} \delta\left(\lambda_{r}\right)
$$

For brevity, let $\delta_{r}$ denote $\delta\left(\lambda_{r}\right)$ and let $W_{r}$ denote the open neighbourhood corresponding to $\lambda_{r}$. Let $g_{r}$ be the inverse of $f$ on $W_{r}$. Define

$$
\begin{aligned}
& \tau_{1}=\delta_{1} \cap \sigma(A), \\
& \tau_{2}=\left(\delta_{2} \backslash \delta_{1}\right) \cap \sigma(A), \\
& \tau_{n}=\left(\delta_{n} \backslash\left[\bigcup_{r=1}^{n-1} \delta_{r}\right]\right) \cap \sigma(A) .
\end{aligned}
$$

Glasgow Math. J. 23 (1982) 171-175. 
Observe that $\left\{\tau_{r}: r=1, \ldots, n\right\}$ is a family of pairwise disjoint sets such that $\tau_{r} \in \Sigma_{p}$,

$$
\begin{aligned}
\tau_{r} & \subseteq \bar{\delta}_{r} \subseteq W_{r} \quad(r=1, \ldots, n), \\
\sigma(A) & \subseteq \bigcup_{r=1}^{n} \tau_{r} .
\end{aligned}
$$

Define

$$
T=\bigoplus_{r=1}^{n} g_{r}\left(A \mid E\left(\tau_{r}\right) X\right)
$$

We know that

$$
\sigma\left(A \mid E\left(\tau_{r}\right) X\right) \subseteq \bar{\tau}_{r} \quad(r=1, \ldots, n) .
$$

Also, by Theorem 14.2 of [2, pp 265-6], $A \mid E\left(\tau_{r}\right) X$ is a prespectral operator with resolution of the identity $E(\cdot) \mid E\left(\tau_{r}\right) X$ of class $\Gamma$.

Now, by Theorem 10.34 of $\left[3\right.$, p. 217], $g_{r}$ is analytic on $W_{r}$, and so it follows from Theorem 5.16 of [2, pp 130-1] that $g_{r}\left(A \mid E\left(\tau_{r}\right) X\right)$ is also prespectral of class $\Gamma$. Also, the resolution of the identity of class $\Gamma$ for $g_{r}\left(A \mid E\left(\tau_{r}\right) X\right)$ is $F_{r}(\cdot)$, where

$$
\begin{aligned}
F_{r}(\delta) & =E\left(g_{r}^{-1}(\delta)\right) \mid E\left(\tau_{r}\right) X \\
& =E\left(g_{r}^{-1}(\delta) \cap \tau_{r}\right) \mid E\left(\tau_{r}\right) X \quad\left(\delta \in \Sigma_{p}, r=1, \ldots, n\right) .
\end{aligned}
$$

Define

$$
F(\delta)=\sum_{r=1}^{n} F_{r}(\delta) \quad\left(\delta \in \Sigma_{p}\right) .
$$

We wish to prove that $T$ is a prespectral operator with resolution of the identity $F(\cdot)$ of class $\Gamma$ and that $f(T)=A$.

Let $\delta_{1}, \delta_{2} \in \Sigma_{p}$. Observe that

$$
\begin{aligned}
F\left(\delta_{1} \cap \delta_{2}\right) & =\sum_{r=1}^{n} F_{r}\left(\delta_{1} \cap \delta_{2}\right) \\
& =\sum_{r=1}^{n} E\left(g_{r}^{-1}\left(\delta_{1} \cap \delta_{2}\right) \cap \tau_{r}\right) \\
& =\sum_{r=1}^{n} E\left(g_{r}^{-1}\left(\delta_{1}\right) \cap \tau_{r} \cap g_{r}^{-1}\left(\delta_{2}\right) \cap \tau_{r}\right) \\
& =\sum_{r=1}^{n} E\left(g_{r}^{-1}\left(\delta_{1}\right) \cap \tau_{r}\right) E\left(g_{r}^{-1}\left(\delta_{2}\right) \cap \tau_{r}\right)
\end{aligned}
$$

It follows that

$$
F\left(\delta_{1} \cap \delta_{2}\right)=\sum_{r=1}^{n} F_{r}\left(\delta_{1}\right) F_{r}\left(\delta_{2}\right)=F\left(\delta_{1}\right) F\left(\delta_{2}\right) \quad\left(\delta_{1}, \delta_{2} \in \Sigma_{p}\right),
$$


using the fact that $\tau_{1}, \ldots, \tau_{n}$ are pairwise disjoint. Also

$$
\begin{aligned}
F(\mathbf{C}) & =\sum_{r=1}^{n} F_{r}(\mathbf{C})=\sum_{r=1}^{n} E\left(g_{r}^{-1}(\mathbf{C}) \cap \tau_{r}\right) \\
& =\sum_{r=1}^{n} E\left(\tau_{r}\right)=E(\sigma(A))=I .
\end{aligned}
$$

Now let $\delta \in \Sigma_{p}$. Observe that

$$
\begin{aligned}
F(\mathbf{C} \backslash \delta) & =\sum_{r=1}^{n} F_{r}(\mathbf{C} \backslash \delta) \\
& =\sum_{r=1}^{n} E\left(g_{r}^{-1}(\mathbf{C} \backslash \delta) \cap \tau_{r}\right) \\
& =\sum_{r=1}^{n}\left[E\left(g_{r}^{-1}(\mathbf{C}) \cap \tau_{r}\right)-E\left(g_{r}^{-1}(\delta) \cap \tau_{r}\right)\right] \\
& =\sum_{r=1}^{n} E\left(g_{r}^{-1}(\mathbf{C}) \cap \tau_{r}\right)-\sum_{r=1}^{n} E\left(g_{r}^{-1}(\delta) \cap \tau_{r}\right) \\
& =I-\sum_{r=1}^{n} E\left(g_{r}^{-1}(\delta) \cap \tau_{r}\right) \\
& =I-F(\delta) .
\end{aligned}
$$

Let $\delta_{1}, \delta_{2} \in \Sigma_{p}$. Then

$$
\begin{aligned}
F\left(\delta_{1} \cup \delta_{2}\right) & =F\left(\mathbf{C} \backslash\left(\mathbf{C} \backslash \delta_{1}\right) \cap\left(\mathbf{C} \backslash \delta_{2}\right)\right) \\
& =I-F\left(\left(\mathbf{C} \backslash \delta_{1}\right) \cap\left(\mathbf{C} \backslash \delta_{2}\right)\right) \\
& =I-F\left(\mathbf{C} \backslash \delta_{1}\right) F\left(\mathbf{C} \backslash \delta_{2}\right) \\
& =I-\left(I-F\left(\delta_{1}\right)\right)\left(I-F\left(\delta_{2}\right)\right) \\
& =F\left(\delta_{1}\right)+F\left(\delta_{2}\right)-F\left(\delta_{1}\right) F\left(\delta_{2}\right) .
\end{aligned}
$$

Also if $\|E(\tau)\| \leq M<\infty$, then clearly $\|F(\tau)\| \leq n M<\infty\left(\tau \in \Sigma_{p}\right)$.

We deduce that if $\left\{\delta_{m}\right\}$ is a pairwise disjoint sequence of sets in $\Sigma_{p}$, then

$$
F\left(\bigcup_{m=1}^{k} \delta_{m}\right)=\sum_{m=1}^{k} F\left(\delta_{m}\right) .
$$

Hence if $x \in X$ and $y \in \Gamma$, then we obtain

$$
\begin{aligned}
\left\langle F\left(\bigcup_{m=1}^{k} \delta_{m}\right) x, y\right\rangle & =\sum_{m=1}^{k}\left\langle F\left(\delta_{m}\right) x, y\right\rangle \\
& =\sum_{m=1}^{k} \sum_{r=1}^{n}\left\langle F_{r}\left(\delta_{m}\right) x, y\right\rangle \\
& =\sum_{m=1}^{k} \sum_{r=1}^{n}\left\langle E\left(g_{r}^{-1}\left(\delta_{m}\right) \cap \tau_{r}\right) x, y\right\rangle \\
& =\sum_{r=1}^{n}\left\langle E\left(g_{r}^{-1}\left(\bigcup_{m=1}^{k} \delta_{m}\right) \cap \tau_{r}\right) x, y\right\rangle .
\end{aligned}
$$


Let $k \rightarrow \infty$. Using the properties of the inverse image and the countable additivity of $\langle E(\cdot) x, y\rangle$ on $\Sigma_{p}$ for $x$ in $X$ and $y$ in $\Gamma$, we deduce that

$$
\begin{aligned}
& \text { (a) } \lim _{k \rightarrow \infty}\left\langle F\left(\bigcup_{m=1}^{k} \delta_{m}\right) x, y\right\rangle \text { exists }(x \in X, y \in \Gamma) \\
& \text { (b) } \lim _{k \rightarrow \infty}\left\langle F\left(\bigcup_{m=1}^{k} \delta_{m}\right) x, y\right\rangle=\left\langle F\left(\bigcup_{m=1}^{\infty} \delta_{m}\right) x, y\right\rangle(x \in X, y \in \Gamma) .
\end{aligned}
$$

This completes the proof that $F(\cdot)$ is a spectral measure of class $\left(\Sigma_{p}, \Gamma\right)$.

Since $g_{r}$ is analytic on a neighbourhood of $\bar{\tau}_{r}$,

$$
g_{r}\left(A \mid E\left(\tau_{r}\right) X\right)=\frac{1}{2 \pi i} \int_{B} g_{r}(\lambda)\left((\lambda I-A) \mid E\left(\tau_{r}\right) X\right)^{-1} d \lambda,
$$

where $B$ is a suitable finite family of contours in $\rho\left(A \mid E\left(\tau_{r}\right) X\right)$. Since $A$ is prespectral, it follows that

$$
A E(\tau)=E(\tau) A \quad\left(\tau \in \Sigma_{p}\right)
$$

and so $A \mid E\left(\tau_{r}\right) X$ commutes with $E(\cdot) \mid E\left(\tau_{r}\right) X$. We deduce readily from this that $F_{r}(\cdot)$ commutes with $g_{r}\left(A \mid E\left(\tau_{r}\right) X\right)$ and consequently

$$
T F(\tau)=F(\tau) T \quad\left(\tau \in \Sigma_{p}\right) .
$$

Also

$$
F(\delta) X=\bigoplus_{r=1}^{n} E\left(g_{r}^{-1}(\delta) \cap \tau_{r}\right) X \quad\left(\delta \in \Sigma_{p}\right)
$$

Each of the subspaces on the right-hand side reduces $T$ and so, by Proposition 1.37 of [2, pp 25-6]

$$
\begin{aligned}
\sigma(T \mid F(\delta) X) & =\bigcup_{r=1}^{n} \sigma\left(T \mid E\left(g_{r}^{-1}(\delta) \cap \tau_{r}\right) X\right. \\
& =\bigcup_{r=1}^{n} \sigma\left(\left(g_{r}\left(A \mid E\left(\tau_{r}\right) X\right) \mid E\left(g_{r}^{-1}(\delta) \cap \tau_{r}\right) X\right.\right. \\
& \subseteq \bar{\delta} \quad\left(\delta \in \Sigma_{p}\right) .
\end{aligned}
$$

It follows that $F(\cdot)$ is a resolution of the identity for $T$. Finally

$$
\begin{aligned}
f(T) & =f\left(\bigoplus_{r=1}^{n} g_{r}\left(A \mid E\left(\tau_{r}\right) X\right)\right) \\
& =\bigoplus_{r=1}^{n}\left(f \circ g_{r}\right)\left(A \mid E\left(\tau_{r}\right) X\right) \\
& =\bigoplus_{r=1}^{n} A \mid E\left(\tau_{r}\right) X \\
& =A
\end{aligned}
$$

and so the proof is complete. 
Corollary. Let $A$, in $L(X)$, be a scalar-type operator of class $\Gamma$. Let $\Omega$ be a region and let $f$ be a function analytic on $\Omega$ such that $\sigma(A) \subseteq f(\Omega)$ and $f^{\prime}(\lambda) \neq 0$ for all $\lambda$ in $\Omega$. Then there is a scalar-type operator $S$ of class $\Gamma$ such that $\sigma(S) \subseteq \Omega$ and $f(S)=A$.

Proof. In the notation of the proof of Theorem $1, A \mid E\left(\tau_{r}\right) X$ is a scalar-type operator of class $\Gamma$ for each $r=1, \ldots, n$. It follows that $g_{r}\left(A \mid E\left(\tau_{r}\right) X\right)$ is also a scalar-type operator of class $\Gamma$ and hence that $T$ is a scalar-type operator of class $\Gamma$. This completes the proof.

Finally, we state the special case of Theorem 1 for spectral operators combined with a result of Apostol [1].

THEOREM 2. Let $A$, in $L(X)$, be a spectral operator. Let $\Omega$ be a region and let $f$ be $a$ function analytic on $\Omega$ such that $\sigma(A) \subseteq f(\Omega)$ and $f^{\prime}(\lambda) \neq 0$ for all $\lambda$ in $\Omega$. Then there is $a$ spectral operator $T_{0}$ on $X$ such that $\sigma\left(T_{0}\right) \subseteq \Omega$ and $f\left(T_{0}\right)=A$. Moreover, if $T \in L(X)$ and $f(T)=A$, then $T$ is a spectral operator.

\section{REFERENCES}

1. C. Apostol, On the roots of spectral operator-valued analytic functions, Rev. Roumaine Math. Pures Appl. 13 (1968), 587-589.

2. H. R. Dowson, Spectral theory of linear operators (Academic Press, 1978).

3. W. Rudin, Real and complex analysis (McGraw Hill, 1966).

Department of Mathematics,

UNIVERSTTY OF GLASGOW.

Present address:

Department of Mathematics

FACULTY OF SCIENCE

KING SAUd UNIVERSITY

RIYADH

Saudi Arabia 